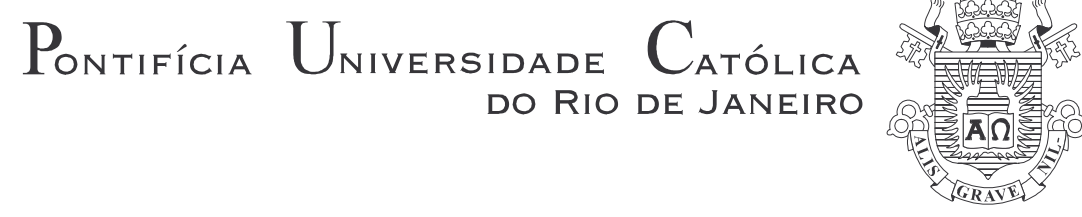

Adriano dos Santos

\title{
Modelo Estocástico para a Exclusão pelo Tamanho Durante o Transporte de Suspensões Particuladas em Meios Porosos
}

Tese apresentada ao Programa de PósGraduação em Engenharia Civil da PUC-Rio como requisito parcial para obtenção do título de Doutor em Ciências de Engenharia Civil: Geotecnia.

Orientadores: Sérgio A. B. da Fontoura Pavel G. Bedrikovetsky

Rio de Janeiro, Setembro de 2005. 


$$
\text { Pontifícia Universidade } \text { Católica }_{\text {do Rio de Janeiro }}
$$

Adriano dos Santos

\title{
Modelo Estocástico para a Exclusão pelo Tamanho Durante o Transporte de Suspensões Particuladas em Meios Porosos
}

Tese apresentada como requisito parcial para obtenção do título de Doutor pelo Programa de Pós-Graduação em Engenharia Civil da PUC-Rio. Aprovada pela Comissão Examinadora abaixo assinada.

\author{
Prof. Sérgio Augusto Barreto da Fontoura \\ Presidente/Orientador \\ Departamento de Engenharia Civil - PUC-Rio \\ Prof. Pavel Bedrikovetsky \\ UENF \\ Co-orientador
}

Prof. Eurípedes do Amaral Vargas Júnior Departamento de Engenharia Civil - PUC-Rio

Prof. Celso Romanel Departamento de Engenharia Civil - PUC-Rio

Dr. Antonio Luiz Serra de Souza CENPES / PETROBRAS

Dra. Rosana Fátima Teixeira Lomba CENPES / PETROBRAS

Dr. Paulo Dore Fernandes CENPES / PETROBRAS

Prof. José Eugênio Leal Coordenador Setorial do Centro Técnico Científico - PUC-Rio 
Todos os direitos reservados. É proibida a reprodução total ou parcial do trabalho sem autorização da universidade, do autor e do orientador.

\section{Adriano dos Santos}

Graduou-se em Física (Bacharelado e Licenciatura) na UFSC (Universidade Federal de Santa Catarina) em 1996. Concluiu o mestrado em Física Estatística na UFSC em 1998. As atuais áreas de interesse são: Transporte em meios porosos, dano à formação, perda de injetividade, modelagem estocástica e recuperação avançada de petróleo.

Ficha Catalográfica

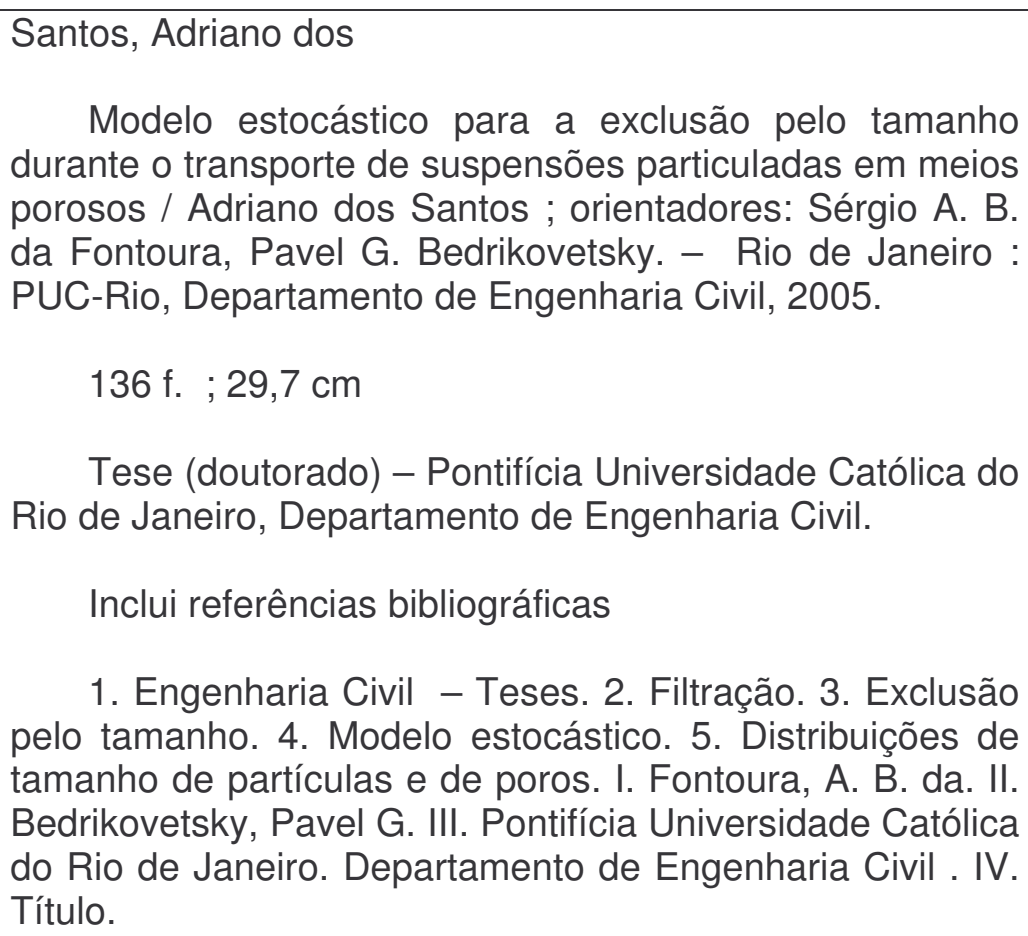

Modelo estocástico para a exclusão pelo tamanho durante o transporte de suspensões particuladas em meios porosos / Adriano dos Santos ; orientadores: Sérgio A. B. da Fontoura, Pavel G. Bedrikovetsky. - Rio de Janeiro : PUC-Rio, Departamento de Engenharia Civil, 2005.

136 f. ; 29,7 cm

Tese (doutorado) - Pontifícia Universidade Católica do Rio de Janeiro, Departamento de Engenharia Civil.

Inclui referências bibliográficas

1. Engenharia Civil - Teses. 2. Filtração. 3. Exclusão pelo tamanho. 4. Modelo estocástico. 5. Distribuições de tamanho de partículas e de poros. I. Fontoura, A. B. da. II. Bedrikovetsky, Pavel G. III. Pontifícia Universidade Católica do Rio de Janeiro. Departamento de Engenharia Civil . IV. Título. 


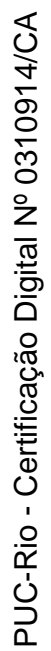

Para os meus pais Walderi e Ângela 


\section{Agradecimentos}

À minha família pelo carinho e incentivo aos estudos.

Aos Professores Dr. Sérgio Fontoura e Dr. Pavel Bedrikovetsky pelo apoio e orientação.

Ao Dr. Antônio Luiz Serra de Souza (CENPES / PETROBRAS), Dr. Farid Shecaira (CENPES / PETROBRAS), Ms. Alexandre Siqueira (CENPES / PETROBRAS), Dr. J. Bruining (Delft University) e Dr. Yannis Yortsos (University of South California) pelas valorosas sugestões para este trabalho.

Aos amigos Adolfo Puime Pires, José Eurico Altoé Junior e Rubens Peres Lopes Junior pelo companheirismo e discussões sobre o conteúdo desta tese.

Ao Ms. Ronaldo Oliveira de Paiva (PETROBRAS) pelos dados fornecidos.

Aos amigos e colegas do GTEP, em especial ao Ewerton, ao Bruno, ao Fredy, à Olga, à Nelly e à Suzana, pelo companheirismo e convívio diário.

À CAPES, à ANP e à FENORTE pelo apoio financeiro concedido durante o meu doutorado.

Ao Diego, Thiago, Luiz Gustavo e Erblai pela amizade e companheirismo.

Aos colegas, professores e funcionários do departamento de Engenharia Civil da PUC-RIO e do Laboratório de Engenharia e Exploração de Petróleo (LENEP/UENF), em especial à Prof. Themis, pelo convívio e apoio. 


\section{Resumo}

dos Santos, Adriano; Fontoura, Sérgio A. B. da; Bedrikovetsky, Pavel G. Modelo estocástico para a exclusão pelo tamanho durante o transporte de suspensões particuladas em meios porosos. Rio de Janeiro, 2005. 136p. Tese de Doutorado - Departamento de Engenharia Civil, Pontifícia Universidade Católica do Rio de Janeiro.

A filtração profunda de suspensões particuladas ocorre em muitos processos industriais e ambientais, como filtração de água e contaminação do solo. Na indústria petrolífera, a filtração profunda ocorre próximo ao poço injetor durante a injeção de água, causando redução de injetividade. A captura de partículas no meio poroso pode ser causada por diferentes mecanismos físicos (exclusão pelo tamanho, forças elétricas, gravidade (sedimentação), etc.). No caso do mecanismo de exclusão pelo tamanho, quanto maiores forem as partículas e menores forem os poros, mais intensa será a captura. Conseqüentemente, maior será o dano à formação. Entretanto, o modelo tradicional não considera as distribuições de tamanho de partículas e de poros. Assumindo que as partículas são capturadas pelo mecanismo de exclusão pelo tamanho, foram deduzidas as equações básicas para o transporte de suspensões particuladas no meio poroso considerando as distribuições de tamanho de poros e de partículas. Apenas o fluxo de água via poros acessíveis transporta partículas, ou seja, as partículas não podem acessar poros menores do que elas. No presente trabalho, os efeitos da redução do fluxo de partículas e da inacessibilidade devido ao fluxo seletivo de diferentes tamanhos de partículas são incluídos no modelo estocástico para a filtração profunda. As soluções analíticas obtidas mostram um comportamento físico mais realístico do que o previsto pelo modelo tradicional. O modelo de medição (concentrações totais) obtido difere substancialmente do modelo tradicional para a filtração profunda. Vários dados experimentais foram tratados, mostrando boa concordância e validando o modelo proposto. Um sistema de equações estocásticas para modelar a formação do reboco externo foi proposto e soluções analíticas foram obtidas, permitindo tratar a filtração profunda e a formação do reboco externo, utilizando o mesmo formalismo matemático.

\section{Palavras-chave}

Filtração; exclusão pelo tamanho; modelo estocástico; distribuições de tamanho de poros e de partículas; dano à formação. 


\section{Abstract}

dos Santos, Adriano; Fontoura, Sérgio A. B. da (Advisor); Bedrikovetsky, Pavel G. (Advisor). Stochastic model for size exclusion mechanism during suspended particle suspension transport in porous medium. Rio de Janeiro, 2005. 136p. DSc. Thesis - Departamento de Engenharia Civil, Pontifícia Universidade Católica do Rio de Janeiro.

Deep bed filtration of water with particles occurs in several industrial and environmental processes like water filtration and soil contamination. In petroleum industry, deep bed filtration occurs near to injection wells during water injection, causing injectivity reduction. It also takes place during well drilling, sand production control, produced water disposal in aquifers, etc. The particle capture in porous media can be caused by different physical mechanisms (size exclusion, electrical forces, bridging, gravity (sedimentation), etc.). In case of size exclusion mechanism, the larger are the particles and the smaller are the pores, the more intensive is the capture and the larger is the formation damage. Nevertheless, the widely used traditional model does not account for particle and pore size distributions. Considering that particles are captured due to size exclusion mechanism, we derived basic equations for transport of particulate suspensions in porous media, accounting for particle and pore radii distributions. Particles are carried by water flowing through the accessible pore space only, i.e. particles cannot access smaller pores. In the current work, the effects of porous space accessibility and particle flux reduction due to selective flow of different size particles are included into the stochastic deep bed filtration model. The particle and pore ensembles for analytical solutions of the derived system show more realistic physics behaviour than that of the traditional model. Averaging of the derived stochastic equations leads to a new deep bed filtration model that significantly differs from the classical deep bed filtration system. Treatment of several experimental data shows good agreement between the laboratory and modelling data and validates the proposed model. The derived stochastic model has been extended to model formation of external filter cake by particles from the injected polydispersed suspension, allowing treating both deep bed filtration and external filter cake formation in the framework of the same system of governing equations. 


\section{Keywords}

Filtration, straining, stochastic model, particle and pore radii distributions, formation damage. 


\section{Sumário}

1 Introdução

2 Revisão bibliográfica $\quad 23$

2.1. Modelo clássico para filtração profunda 23

2.2. Modelo estocástico na micro-escala 27

$\begin{array}{ll}\text { 2.3. Modelos de rede } & 28\end{array}$

2.4. Modelo de reboco com tempo de transição 29

2.5. Modelo multicomponente para filtração e formação do reboco 30

3 Modelo estocástico na micro escala com efeitos de acessibilidade e redução de fluxo de partículas 33

3.1. Modelo geométrico para o meio poroso 33

3.2. Dedução das equações básicas 35

3.3. Problema de Goursat: dinâmica de captura de partículas e bloqueio de poros na face de entrada do meio poroso 46

4 Soluções analíticas para o modelo proposto 48

4.1. Meio poroso com único tamanho de poro 48

4.1.1. Solução analítica $\quad 49$

4.1.2. Dados experimentais 53

4.1.3. Modelo de medição (concentrações totais) 54

4.1.4. Discussão sobre os coeficientes de redução de fluxo e de acessibilidade $\quad 56$

4.2. Rocha constituída de poros com pequena variação de tamanho 58

4.2.1. Solução analítica $\quad 59$

4.2.2. Comparação com dados experimentais 66

4.2.3. Comprimento de penetração 70

4.2.4. Modelo de medição (concentrações totais) 72

4.3. Injeção de suspensões com baixa concentração 74

$\begin{array}{ll}\text { 4.3.1. Solução analítica } & 74\end{array}$ 
5 Modelo efetivo (macro escala) para filtração profunda considerando os fatores de redução de fluxo e de acessibilidade

5.1. As funções de acessibilidade e redução de fluxo para injeção de partículas de tamanho intermediário em uma rocha contendo poros de dois tamanhos

5.2. Injeção de partículas de tamanho único em uma rocha contendo diferentes tamanhos de poros

5.3. - Tratamento de dados experimentais relacionados à redução de permeabilidade

5.4. Obtenção de um modelo efetivo a partir das equações estocásticas

6 Modelo analítico para filtração profunda com redução de fluxo e acessibilidade

6.1. Caso onde os fatores de acessibilidade e de redução de fluxo são constantes

6.1.1. Determinação experimental dos coeficientes do modelo

7 Modelo transiente para a formação do reboco externo

7.1. Distribuição de raio de poros e crescimento do reboco externo 


\section{Lista de figuras}

Figura 1: Esquema mostrando a injeção de água num reservatório de petróleo.

Figura 2: Mecanismos de captura de partículas: (A) Exclusão pelo tamanho (i) e "bridging" (ii); (B) deposição; (C) movimento browniano de uma partícula sobre uma linha de fluxo.

Figura 3: Relação entre o coeficiente de filtração $\lambda_{0}$ e o parâmetro $\eta$ (razão entre o raio médio das partículas e o raio médio dos poros). Neste gráfico estão representados os resultados para partículas sólidas (losangos), líquidas (quadrados) e uma mistura de ambas (triângulos) (Bedrikovetsky et al., 2001). 26 Figura 4: Esquema mostrando a captura de partículas grandes em poros menores do que elas.

Figura 5: Meio poroso constituído por um conjunto de capilares intersecionados por câmaras de mistura: (a) trajetória das partículas nos capilares e nas câmaras de mistura, (b) seção transversal ao fluxo, (c) esquema mostrando a ligação entre poros de conjuntos subseqüentes de capilares.

Figura 6: Esquema mostrando as condições iniciais e de contorno

Figura 7: Evolução das concentrações C, $\Sigma$ e H.

Figura 8: Distribuições de poros e de partículas em suspensão em um meio com tamanho único de poros.(a) condições iniciais e de contorno para as distribuições de concentração de poros e de partículas em suspensão. (b) distribuição de concentração de partículas para qualquer $\mathrm{X}$ e $\mathrm{T}$ (curva sólida) e para $\mathrm{X}=0$ (curva pontilhada); distribuição de concentração de poros na face de entrada do meio poroso para $\mathrm{T}>0$.

Figura 9: Curvas para o tempo de chegada ("breakthrough time") para diferentes tamanhos de partículas (em $\mathrm{X}=1$ ): (1) - para partículas menores que $\mathrm{r}_{\mathrm{p}}{ }^{\prime}$ (de acordo com o modelo proposto); (2) - para partículas maiores que $\mathrm{r}_{\mathrm{p}}{ }^{\prime}$ (de acordo com o modelo proposto); (3) - para partículas maiores que $r_{p}{ }^{\prime}$ (ignorando os fatores de acessibilidade e de redução de fluxo).

Figura 10: Imagem de microscopia eletrônica (aumento de 200x) das esferas que 
constituem o meio poroso (Tufenkji et al., 2004).

Figura 11: Concentração de partículas em suspensão na saída $(X=1)$ do meio poroso (Tufenkji et al., 2004).

Figura 12: Distribuições de tamanho de poros e de partículas em suspensão em um meio poroso com pequena variação no tamanho de poros: (a) condições iniciais e de contorno para as distribuições de poroso e de partículas em suspensão; (b) distribuições de partículas em suspensão atrás da "frente de concentração" para T >0 (curva sólida), concentração injetada (curva tracejada) e concentração de poros.

Figura 13: Velocidade da frente de deslocamento de uma população de partículas em função do seu raio.

Figura 14: Perfis de distribuição de concentração para partículas de tamanho intermediário durante a filtração em um meio poroso com pequena variação no tamanho de poros. As linhas 1, 2 e 3 correspondem a diferentes populações de partículas $\left(\mathrm{r}_{\mathrm{s} 1}<\mathrm{r}_{\mathrm{s} 2}<\mathrm{r}_{\mathrm{s} 3}\right)$.

Figura 15: Distribuição de concentração de partículas em suspensão na saída do meio poroso. A linha (1) corresponde à concentração de partículas pequenas $\left(\mathrm{r}_{\mathrm{s}}<\right.$ $\mathrm{r}_{\mathrm{pmin}}$ ); a linha (2) está relacionada com a concentração de partículas com tamanho intermediário $\left(\mathrm{r}_{\mathrm{pmin}}<\mathrm{r}_{\mathrm{s}}<\mathrm{r}_{\mathrm{pmax}}\right)$ e a linha (3) corresponde à concentração de partículas grandes $\left(\mathrm{r}_{\mathrm{s}}>\mathrm{r}_{\mathrm{pmin}}\right)$.

Figura 16: Perfis de densidade de concentração para diferentes tamanhos de partículas. Cada frente se desloca com velocidade $\alpha\left(r_{s}\right) / \gamma\left(r_{s}\right)$. As linhas 1 e 4 correspondem às partículas pequenas $\left(\mathrm{r}_{\mathrm{s} 1}<\mathrm{r}_{\mathrm{pmin}}\right)$ e grandes $\left(\mathrm{r}_{\mathrm{s} 4}>\mathrm{r}_{\mathrm{pmax}}\right)$, respectivamente. As linhas 2 e 3 estão relacionadas às partículas de tamanho intermediário $\left(\mathrm{r}_{\mathrm{s} 2}<\mathrm{r}_{\mathrm{s} 3}\right)$.

Figura 17: Concentrações normalizadas na saída do meio poroso $(X=1)$ para a injeção de tamanhos crescentes de partículas $(0.32 \mu \mathrm{m}, 1.0 \mu \mathrm{m}, 1.9 \mu \mathrm{m}$ e $4.1 \mu \mathrm{m})$ suspensas em água deionizada em um meio poroso $(\phi=0.43)$ constituído de grãos de quartzo com diâmetro médio igual à $0.21 \mathrm{~mm}$ (Tufenkji et al., 2004).

Figura 18: Distribuição acumulada do diâmetro dos grãos de quartzo que constituem o meio poroso (Tufenkji et al., 2004).

Figura 19: Modelo geométrico adotado para determinar o raio dos grãos, a partir do qual o mecanismo de exclusão pelo tamanho torna-se desprezível (Herzig et 
al., 1970).

Figura 20: Imagens de microscopia eletrônica dos grãos de quartzo. (a) aumento de 500x e (b) aumento de 2000x (Tufenkji et al., 2004).

Figura 21: Efeito do tamanho das partículas no comprimento máximo de penetração $\left\langle X\left(r_{s}\right)\right\rangle_{\max }$ durante o processo de filtração em um meio poroso com pequena variação de tamanho de poros.

Figura 22: Distribuições de concentração de poros e de partículas em suspensão durante o processo de filtração profunda: (a) distribuições iniciais; (b) distribuições atrás da frente de deslocamento para $\mathrm{T}>0$.

Figura 23: Distribuições de tamanho de partículas na face de injeção e distribuição inicial de tamanho de poros $\mathrm{f}_{\mathrm{p}}\left(\mathrm{r}_{\mathrm{p}}, \mathrm{X}, 0\right)$.

Figura 24: Perfis de concentração de partículas suspensas de diferentes tamanhos $\left(\mathrm{r}_{\mathrm{s}}=11.5 \mu \mathrm{m}, 12.5 \mu \mathrm{m}\right.$ e $\left.13.5 \mu \mathrm{m}\right)$ para tempos maiores do que o tempo de chegada $\left(\mathrm{T}_{\mathrm{br}}\right)$.

Figura 25: Perfis de concentração de partículas capturadas de diferentes tamanhos $\left(\mathrm{r}_{\mathrm{s}}=12 \mu \mathrm{m}, 13 \mu \mathrm{m}\right.$ e $\left.13.5 \mu \mathrm{m}\right)$ para $\mathrm{T}=1.2 \times 10^{4}$ pvi.

Figura 26: Perfis de concentração total de partículas retidas para os tempos $1.2 \times$ $10^{3}, 6 \times 10^{3}$ e $1.2 \times 10^{4}$ pvi.

Figura 27: Distribuição de concentração de poros na face de entrada do meio poroso $\mathrm{H}\left(\mathrm{r}_{\mathrm{p}}, 0, \mathrm{~T}\right)$ para os tempos $0,6.7 \times 10^{3}, 1.67 \times 10^{4}$ e $2 \times 10^{5}$ pvi (curvas $1,2,3 \mathrm{e}$ 4 , respectivamente).

Figura 28: Distribuição de concentração de poros na face de entrada do meio poroso $\mathrm{H}\left(\mathrm{r}_{\mathrm{p}}, 0, \mathrm{~T}\right)$ para os tempos $0,6.7 \times 10^{3}, 1.67 \times 10^{4} \mathrm{e} 2 \times 10^{5}$ pvi (curvas $1,2,3 \mathrm{e}$ 4 , respectivamente).

Figura 29: Queda de permeabilidade em função do tempo adimensional.

Figura 30: Gráfico do fator de redução de fluxo como função do fator de acessibilidade para $\mathrm{r}_{\mathrm{p} 2} / \mathrm{r}_{\mathrm{p} 1}=2$.

Figura 31: Fatores de acessibilidade e de redução de fluxo como funções da concentração de partículas capturadas para $\mathrm{r}_{\mathrm{p} 2} / \mathrm{r}_{\mathrm{p} 1}=2$.

Figura 32: Distribuição de raios de poros e de partículas para o caso da injeção de partículas de tamanho único em uma rocha com poros de tamanhos discretos $\left(\mathrm{r}_{\mathrm{p} 1}\right.$, $\left.\mathrm{r}_{\mathrm{p} 2}, \ldots, \mathrm{r}_{\mathrm{pN}}\right)$.

Figura 33: (a) Microscopia de varredura (Scanning Electron Microscopy - SEM) 
mostrando a superfície da membrana utilizada no experimento 1. (b) modelagem da membrana mostrada na figura (a) (Seminario et al., 2002).

Figura 34: (a) Microscopia de varredura mostrando a superfície da membrana utilizada no experimento 2. (b) Representação da membrana mostrada na figura (a) (Seminario et al., 2002).

Figura 35: Distribuição de tamanho de poros da membrana utilizada na modelagem do experimento 1 (ver Figura 33b) (Seminario et al., 2002). 98 Figura 36: Distribuição de tamanho de poros da membrana utilizada na modelagem do experimento 2 (ver Figura 34b) (Seminario et al., 2002). Figura 37: Distribuição de tamanho de partículas em suspensão no fluido injetado (Seminario et al., 2002).

Figura 38: Coeficientes de redução de fluxo $\alpha$ e de acessibilidade $\gamma$ em função da concentração de partículas capturadas $\sigma$ para 5 e 9 tamanhos distintos de poros 102 Figura 39: Comparação entre a redução de permeabilidade obtida experimentalmente por Seminario et al.(2002) e a prevista pelo modelo proposto (considerando 2, 5 e 9 tamanhos distintos de poros) para a membrana apresentada na Figura 33. Neste caso, o coeficiente de filtração $\lambda^{\prime}$ utilizado no ajuste é igual a $2.3 \mathrm{~m}^{-1}$ e o raio efetivo das partículas $\left(\mathrm{r}_{\mathrm{s}}{ }^{\prime}\right.$ ) foi considerado igual à $2.1 \mu \mathrm{m} . \quad 103$ Figura 41: Comparação entre a queda de permeabilidade obtida experimentalmente (Seminario et al.,2002) e a prevista pelo modelo (considerando 2, 5 e 9 tamanhos distintos de poros) para a membrana mostrada na Figura 34. Neste caso, o coeficiente de filtração $\lambda^{\prime}$ utilizado no ajuste é igual a $100 \mathrm{~m}^{-1}$ e o raio efetivo das partículas $\left(\mathrm{r}_{\mathrm{s}}{ }^{\prime}\right)$ foi considerado igual à $0.4 \mu \mathrm{m} . \quad 104$ Figura 42: Distribuições de tamanho de poros e de partículas. As áreas $c_{1}$ e $c_{3}$ representam a porção de partículas que não participam do processo de filtração profunda (partículas grandes e pequenas, respectivamente).

Figura 43: Perfil de concentração de partículas em suspensão. A frente se move com velocidade $\alpha / \gamma$.

Figura 44: Concentração de partículas na saída do meio poroso $(X=1)$. As linhas (1), (2) e (3) correspondem as concentrações $c_{1}, c_{2}$ e $c_{3}$, respectivamente (ver Figura 42).

Figura 45: Concentração de partículas em suspensão na saída do meio poroso (AlAbduwani et al., 2005). 
Figura 46: Perfis de deposição $(\sigma(X)$, em unidades HU) ao longo da amostra para vários tempos. A primeira curva (de baixo para cima) corresponde ao tempo $\mathrm{T}=19.7$ pvi. As curvas subseqüentes são para os tempos 189, 367, 646, 927, 1500, 2090, 2920, 3770 e 4560 pvi (Al-Abduwani et al., 2005).

Figura 47: Variação da impedância em função do tempo adimensional (volumes porosos injetados, pvi) segundo Al-Abduwani et al. (2005).

Figura 48: Concentração de partículas em suspensão na interface reboco externo e meio poroso $(\mathrm{x}=0)$ em função do tempo.

123

Figura 49: Perfis de concentração para um tempo $t_{1}$ menor que o tempo de transição $\left(t_{1}<t_{t r}\right)$ e dois tempos $t_{2}$ e $t_{3}$ maiores que o tempo de transição $\left(t_{t r}<t_{2}<\right.$ $\left.t_{3}\right)$.

125

Figura 50: Concentração de partículas em suspensão na saída da amostra $(\mathrm{x}=\mathrm{L})$.

Figura 51: Perfis de concentração de partículas capturadas para diversos tempos $\left(\mathrm{t}_{1}<\mathrm{t}_{2}<\mathrm{t}_{3}<\mathrm{t}_{\mathrm{tr}}<\mathrm{t}_{4}<\mathrm{t}_{5}\right)$. 


\section{Nomenclatura}

$f \quad$ função de distribuição de probabilidade (PDF), $\mathrm{L}^{-1}$

$c \quad$ concentração total de partículas em suspensão, $\mathrm{L}^{-3}$

C distribuição de concentração de partículas em suspensão, $\mathrm{L}^{-4}$

$f_{\mathrm{s}} \quad$ função de distribuição de tamanho de partículas, $\mathrm{L}^{-1}$

$f_{\mathrm{T}} \quad$ distribuição de tamanho de partículas capturadas, $\mathrm{L}^{-1}$

$\underline{f_{T}}$ distribuição de tamanho de partículas capturadas em poros de raio $r_{\mathrm{p}}$, $\mathrm{L}^{-2}$

$h \quad$ concentração total de poros, $\mathrm{L}^{-3}$

$H$ distribuição de concentração de poros, $\mathrm{L}^{-4}$

$f_{\mathrm{p}} \quad$ distribuição de tamanho de poros, $\mathrm{L}^{-1}$

$j \quad$ fluxo de partículas de raio $r_{\mathrm{s}}$ por unidade de área da seção transversal,

$\mathrm{L}^{-3} \mathrm{~T}^{-1}$

$\underline{j} \quad$ distribuição de fluxo de partículas de raio $r_{s}$ através de poros de raio $r_{p}$

por unidade de área da seção transversal, $\mathrm{L}^{-4} \mathrm{~T}^{-1}$

$J(T) \quad$ impedância

$k_{o} \quad$ permeabilidade inicial, $\mathrm{L}^{2}$

$k(\sigma) \quad$ função de dano à formação

L comprimento do meio poroso, $\mathrm{L}$

$p \quad$ pressão, $\mathrm{M} / \mathrm{T}^{2} \mathrm{~L}$

$P \quad$ probabilidade de uma partícula com raio $r_{\mathrm{s}}$ encontrar um poro com raio $r_{\mathrm{p}}$

$r_{p} \quad$ raio do poro, $\mathrm{L}$

$r_{s} \quad$ raio da partícula, $\mathrm{L}$

$s \quad$ desvio padrão, $\mathrm{L}$

$t$ tempo dimensional, $\mathrm{T}$

$T \quad$ tempo adimensional

$U \quad$ velocidade superficial do fluido, $\mathrm{L} / \mathrm{T}$

$x \quad$ coordenada linear, $\mathrm{L}$

$X \quad$ coordenada linear adimensional

$\langle x\rangle \quad$ comprimento médio de penetração, $\mathrm{L}$ 
$\alpha \quad$ fator de redução de fluxo

$\beta \quad$ coeficiente de dano à formação

$\delta \quad$ função delta de Dirac's

$\gamma \quad$ fator de acessibilidade

$\phi \quad$ porosidade

$\lambda^{\prime} \quad$ coeficiente de filtração dimensional, $\mathrm{L}^{-1}$

$\lambda \quad$ coeficiente de filtração adimensional

$\mu \quad$ viscosidade, $\mathrm{ML}^{-1} \mathrm{~T}^{-1}$

$\underline{\Sigma}\left(r_{s}, r_{p}\right) \quad$ distribuição de concentração para partículas de raio $r_{\mathrm{s}}$ capturadas em poros com raio $r_{\mathrm{p}}, \mathrm{L}^{-5}$

$\Sigma\left(r_{s}\right) \quad$ distribuição de concentração de partículas com raio $r_{\mathrm{s}}$ retidas, $\mathrm{L}^{-4}$

$\sigma \quad$ concentração total de partículas capturadas, $\mathrm{L}^{-3}$

\section{Subscritos e superescritos}

$0 \quad$ valor inicial $(T=0)$

br chegada da frente de deslocamento (breakthrough)

c reboco externo (cake)

f frente de deslocamento

p poro

s partículas em suspensão

$\operatorname{tr} \quad$ transição

T partículas capturadas

(0) valor no contorno $(X=0)$ 